\title{
Ribokinase screened from T7 phage displayed Mycobacterium tuberculosis genomic DNA library had good potential for the serodiagnosis of tuberculosis
}

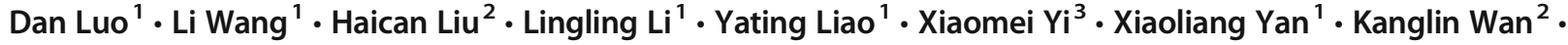 \\ Yanhua Zeng ${ }^{1}$
}

Received: 22 January 2019 / Revised: 5 March 2019 / Accepted: 7 March 2019 / Published online: 8 May 2019

(C) Springer-Verlag GmbH Germany, part of Springer Nature 2019

\begin{abstract}
Tuberculosis caused by Mycobacterium tuberculosis (M. tuberculosis) is the leading cause of death among infectious diseases in the worldwide. Lack of more sensitive and effective diagnostic reagents has increased the awareness of rapid diagnosis for tuberculosis. In this study, T7 phage displayed genomic DNA library of M. tuberculosis was constructed to screen the antigens that specially bind with TB-positive serum from the whole genome of M. tuberculosis and to improve the sensitivity and specificity of tuberculosis serological diagnosis. After three rounds of biopanning, results of DNA sequencing and BLAST analysis showed that 19 positive phages displayed four different proteins and the occurrence frequency of the phage which displayed ribokinase was the highest. The results of indirect ELISA and dot immunoblotting indicated that representative phages could specifically bind to tuberculosis-positive serum. The prokaryotic expression vector containing the DNA sequence of ribokinase gene was then constructed and the recombinant protein was expressed and purified to evaluate the serodiagnosis value of ribokinase. The reactivity of the recombinant ribokinase with different clinical serum was detected and the sensitivities and specificities in tuberculosis serodiagnosis were $90 \%$ and $86 \%$, respectively by screening serum from tuberculosis patients $(n=90)$ and uninfected individuals $(n=90)$ based on ELISA. Therefore, this study demonstrated that ribokinase had good potential for the serodiagnosis of tuberculosis.
\end{abstract}

Keywords Ribokinase $\cdot$ T7 phage displayed genomic DNA library $\cdot$ Mycobacterium tuberculosis $\cdot$ Serological diagnosis

Dan Luo and Li Wang contributed equally to this work.

Kanglin Wan

wankanglin@icdc.cn

Yanhua Zeng

zengyihua21cn@126.com

Dan Luo

2239269298@qq.com

Li Wang

1041736494@qq.com

Haican Liu

liuhaican@126.com

Lingling Li

605782805@qq.com

Yating Liao

1540768354@qq.com
Xiaomei Yi

811233206@qq.com

Xiaoliang Yan

1173704988@qq.com

1 Institute of Pathogenic Biology, Hengyang Medical College, Hunan Provincial Key Laboratory for Special Pathogens Prevention and Control, Hunan Province Cooperative Innovation Center for Molecular Target New Drug Study, University of South China, Hengyang 421001, People's Republic of China

2 State Key Laboratory for Infectious Disease Prevention and Control/ National Institute for communicable Disease Control and Prevention, Chinese Center for Disease Control and Prevention, Beijing 102206, People's Republic of China

3 Reproductive Medical Center, The Affiliated First Hospital, University of South China, Hengyang 421001, People's Republic of China 


\section{Introduction}

Tuberculosis (TB), the chronic infectious disease caused by M. tuberculosis remains an important public health problem globally (Xu et al. 2012). The main causes of morbidity and mortality were the emergence of antibiotic resistant strains and HIV coinfection (Krishna et al. 2016; Machado et al. 2018). The World Health Organization claimed that there were about average 8.5 million incident cases and 1.5 million deaths of TB all over the world per year during the past 3 years (You et al. 2017). The lack of rapid and sensitive diagnostic methods leaded to much misdiagnose of TB. Diagnosing and treating latent TB infection is an important strategy to reduce and eliminate the prevalence of TB.

Over the past decades, important progress has been made in controlling the spread of TB. Direct sputum smear microscopy is most commonly used to diagnose active TB. Sputum smear microscopy is simple, fast, and reliable but with poor sensitivity. Sputum sample culture is the gold standard for the diagnosis of TB, which cannot identify individuals with latent TB infection (Deng et al. 2018; Zhou et al. 2015). Traditional tuberculin skin test (TST) is non-specific due to antigen crossreactivity between $M$. bovis BCG vaccine strain and environmental mycobacteria (Roupie et al. 2018). Regardless of the advantages of rapidness, sensitivity, and specificity, advanced molecular technique such as PCR is costly and not available everywhere (Theron et al. 2011). However, serological diagnosis is a simple, rapid, and inexpensive, but effective method for in vitro diagnosis of TB and has become a hot topic in TB diagnosis in recent years (Baumann et al. 2014; Yan et al. 2018).

Phage display technology established by Smith GP in 1985 has been widely used in therapeutics, diagnostics, vaccine development, and epitope mapping (Qiang et al. 2017; Wang and Wang 2017). Previous studies demonstrated the potential of page display technology for antigen discovery by identifying M. pneumoniae polypeptides containing B cell epitopes, which recognized by immunoglobulins of infected individuals (Beghetto et al. 2009). This technology has already shown great efficacy in identifying novel antigens from human pathogens such as Toxoplasma gondii (Di Cristina et al. 2004) and Streptococcus pneumoniae (De Paolis et al. 2007). Our previous study also demonstrated that two mimotope peptides screened from phage display peptide library have potential serodiagnosis value for TB (Wang et al. 2016).

In the present study, we reported that ribokinase (RK) was screened from T7 page displayed M. tuberculosis genome DNA library using TB-positive serum primarily purified by saturated ammonium sulfate as the target molecule, and the reactivates of RK with different clinical serum samples were detected. The specificity and sensitivity were analyzed to evaluate the potential serodiagnosis value for TB. Results in this study exhibited that RK screened from T7 phage display M. tuberculosis genome DNA library had good specificity and sensitivity for the serodiagnosis of TB. Thus, RK is a promising antigen for the development of rapid, highly sensitive, and specific serological diagnosis agents of TB.

\section{Materials and methods}

\section{Clinical data of patients}

A total of 90 TB-positive sera were from patients (age range, 1-85 years old) suffered from TB diagnosed using the sputum smear culture and X-ray inspection, and the antibody to $M$. tuberculosis were all positive by indirect ELISA and 90 TB-negative sera were from healthy controls (age range, 20-63 years old). The criteria for the healthy control included no TB contact history and BCG vaccination, no clinical signs or symptoms of $\mathrm{TB}$, the sputum smear culture and TST test were negative, normal chest X-ray, and the antibody to M. tuberculosis were negative by indirect ELISA (Luo et al. 2017). All these sera were from the Third People's Hospital, the specialized TB hospital, of Hengyang City in China. Prior written and informed consent were obtained from every participant and this study was approved by the ethics review board of University of South China.

\section{Construction of the T7 phage displayed M. tuberculosis genomic DNA library}

Construction of the T7 phage displayed M. tuberculosis genomic DNA library was performed as previously described by Talwar et al. (2015). The genomic DNA of M. tuberculosis H37Rv was extracted and donated by strain Chinese Center For Disease Control And Prevention (CDC, Beijing, China). The genomic DNA of M. tuberculosis H37Rv was extracted using CTAB (Cetyl trimethyl ammonium bromide) method as described by Yang et al. (2011). The genomic DNA of M. tuberculosis was digested by EcoR I and Hind III and then joined to the T7 10-3b vector using T4 DNA ligase. The library was packaged in vitro using the T7 Select system kit (Novagen,70580-3) as described by manufacturer's instructions. The recombinant T7 phages were then transformed into BLT5403 to construct the T7 phage displayed genomic DNA library. To evaluate the quality of the library, the titer was determined and the randomness was analyzed using $5^{\prime}-$ GGAGCTGT CGTATTCCAGTC- $3^{\prime}$ and 5'-AACCCCTCAAGACC CGTTTA-3' as specific upstream and downstream primers, respectively, to amplify the exogenous DNA sequence of randomly selected 16 phage plaques by PCR. 


\section{Biopanning of the T7 phage displayed M. tuberculosis genomic DNA library}

The biopanning of the $\mathrm{T} 7$ phage displayed M. tuberculosis genomic DNA library was implemented as described by Sakamoto et al. (2016). Briefly, the collected TB-positive serum was preliminary purified by saturated ammonium sulfate precipitation method as described by Wang and Wang (2017). The protein concentration of the purified mixed serum was measured by the quantitative method of Bicinchoninic acid (BCA) protein assay. Three rounds of biological panning were then performed for the constructed T7 phage displayed M. tuberculosis genomic DNA library using the purified mixed TB-positive serum as target molecule. Briefly, for the first round of panning, ELISA plates were coated with $100 \mu \mathrm{L}$ of purified serum $(100 \mu \mathrm{g} / \mathrm{mL})$ overnight at $4{ }^{\circ} \mathrm{C}$ and then blocked for $2 \mathrm{~h}$ at $4{ }^{\circ} \mathrm{C}$ using $5 \mathrm{mg} / \mathrm{mL}$ bovine serum albumin (BSA) in $\mathrm{NaHCO} 3(0.1 \mathrm{~mol} / \mathrm{L}, \mathrm{pH}$ 8.6). And the plates were then incubated with the amplified phage display peptide library $\left(3.0 \times 10^{11}\right.$ phages) diluted in $100 \mu \mathrm{L}$ PBST with slow rotation (60 $\mathrm{min}$ at room temperature). After the unbound phages were discarded by washing 6 times with PBST, the bound phage were eluted with $0.2 \mathrm{M}$ Glycine- $\mathrm{HCl}$ ( $\mathrm{pH} 2.2$ ) by gentle shaking for $10 \mathrm{~min}$, and then neutralized with $1 \mathrm{M}$ Tris- $\mathrm{HCl}$ ( $\mathrm{pH}$ 9.1). The reverse adsorption was then performed using an empty ELISA plate and negative serum respectively to exclude non-specific binding phages. The basic steps of the second and third round of biopanning were similar to those of the first round except for decreasing target protein concentration to $60 \mu \mathrm{g} / \mathrm{mL}$, as well as increasing the concentration of Tween 20 to $0.3 \%$ in PBST elution and reducing the incubation time to $30 \mathrm{~min}$ or prolonging the elution time to $20 \mathrm{~min}$. Finally, the yield of each round of biopanning was calculated based on the output and the input of phages.

\section{DNA sequencing and BLAST analysis}

The phages challenged by three cycles of biopanning were expanded and cultured. Then, 19 colonies were randomly selected from the plates with less than 100 plaques and exogenous DNA fragments were amplified by PCR using $5^{\prime}-$ GGAGCTGTCGTA TTCCAGTC- $3^{\prime}$ and 5'-AACCCCTC AA GACCCGTTTA-3' as the specific upstream and downstream primers, respectively. The PCR products were analyzed by $1.5 \%$ agarose gel electrophoresis and the exogenous DNA sequences displayed by phages were sequenced and then BLAST alignment were performed.

\section{Analysis of the representative phages}

The reactivities of representative phages with TB-positive serum was monitored by phage ELISA and Dot immunobinding assay respectively as described by Shi et al. (2018). Briefly, for the phage ELISA, purified TB-positive serum was coated onto an ELISA plate and blocked with $0.5 \%$ BSA for $1 \mathrm{~h}$ at room temperature. One-microliter purified phages (approximately $10^{11}$ phages) were added to the plate and incubated for $2 \mathrm{~h}$ at ambient temperature. After rinsing with $0.5 \%$ PBST for five times, $200 \mu \mathrm{L}$ of 1:3000 diluted HRP-labeled anti-T7 pIII monoclonal antibody (NEB, Beverly, MA, USA) was added and incubated at $37^{\circ} \mathrm{C}$ for $15 \mathrm{~min}$, followed by an additional 6 times washes with PBST. And the color reaction was observed using TMB solution as a substrate for $15 \mathrm{~min}$ at $37{ }^{\circ} \mathrm{C}$. The reaction was interrupted by $\mathrm{H}_{2} \mathrm{SO}_{4}(2 \mathrm{M})$ and the optical density (OD) values were detected at $450 \mathrm{~nm}$ using a microplate reader (Tecan, Infinite F50, Swiss).

For dot immunobinding assay, the small grids were marked on PVDF membrane soaked with methanol. One microliter of each representative phage (approximately $10^{11}$ phages) was tested on each grid. The purified TB-positive serum was used as positive control. BSA, TB-negative serum and wild type T7 phage were used as the corresponding controls. The PVDF membrane was then dried at room temperature followed by blocking overnight at $4{ }^{\circ} \mathrm{C}$. The membrane was washed 6 times using PBST. The first antibody (mixed TB-positive serum diluted 1:200 with PBS) was taken to soak the film for $2 \mathrm{~h}$ at $37{ }^{\circ} \mathrm{C}$ followed by washing 6 times. The samples were incubated with 1:5000 diluted HRP-labeled goat anti-human IgG (Cat \#62-8420, Invitrogen, USA) at room temperature for $1 \mathrm{~h}$. The membrane was then rinsed 6 times with PBST. Finally, the color reaction was produced with Electrochemiluminescence (ECL) and measured using a chemiluminescence imaging system.

\section{Expression and purification of RK}

According to the results of BLAST analysis, the inserted exogenous DNA sequence (153 bp) for representative phage P1 was $100 \%$ homologous with the DNA sequence of the 436 588 of the $R K$ gene. Therefore, the recombinant RK was expressed and purified to further study the potential value of RK in the serodiagnosis of TB. M. tuberculosis genomic DNA extracted using CTAB was used as a template, ATGGAT CCCTAGGCCAGCACGGCGA and ATCTCGAGGTGA CGATCGCGGTAACCG were used as specific upstream and downstream primers, and Bam HI and Xho I enzyme cleavage site were introduced, respectively. The amplification profile was $94{ }^{\circ} \mathrm{C}$ for $10 \mathrm{~min}, 30$ cycles consisting of $94^{\circ} \mathrm{C}$ for $50 \mathrm{~s}, 66^{\circ} \mathrm{C}$ for $1 \mathrm{~min}$ and $72{ }^{\circ} \mathrm{C}$ for $1 \mathrm{~min}$ followed by a $72{ }^{\circ} \mathrm{C}$ final extension for $10 \mathrm{~min}$. Escherichia coli Rosetta (DE3) and the prokaryotic expression vector $p E T-28 a$ (Novagen, Madison, WI, USA) were previously conserved in our laboratory. The amplification product was purified and cloned to the prokaryotic expression vector $p E T-28 a(+)$. And then the recombinant vector $p E T-28 a(+)-R K$ was transformed into Escherichia coli Rosetta and then the recombinant bacteria 
was induced with $1 \mathrm{mM}$ isopropyl- $\beta-\mathrm{D}$-galactopyranoside (IPTG) at $27^{\circ} \mathrm{C}$ for $8 \mathrm{~h}$ at $180 \mathrm{rpm}$ to express the recombinant protein. The expressed protein was analyzed by $12 \%$ agarose gel electrophoresis. In order to purify the recombinant protein, IPTG-induced recombinant bacteria were harvested and lysed and then centrifuged at $13000 \mathrm{rpm}$ for $30 \mathrm{~min}$. The supernatant was incubated with pre-equilibrated Ni-NTA beads (Qiagen, Inc., Germany) and then washed using buffer A at different $\mathrm{pH}(8.4,8.2,8.0,7.8,7.4,6.3$, respectively) and eluted with buffer A ( $\mathrm{pH}$ 8.2). The protein concentration was detected using bicinchoninic acid protein assay kit (Pierce, Rockford, USA).

\section{Identification of recombinant protein by Western blot}

The expressed recombinant protein was boiled for $10 \mathrm{~min}$ at $100{ }^{\circ} \mathrm{C}$ in SDS-loading buffer. The sample was subjected to SDS-PAGE electrophoresis on $12 \%$ acrylamide gel. Protein samples were transferred onto PVDF membranes using semidry transfer unit (Bio-Rad, Corning Inc., NY, USA) and the membranes were then blocked at $37^{\circ} \mathrm{C}$ water-jacketed incubator for $2 \mathrm{~h}$ with $5 \%(w / v)$ skim milk diluted in TBST. After being washed three times with TBST, PVDF membranes were incubated for $2 \mathrm{~h}$ at $37{ }^{\circ} \mathrm{C}$ with mouse anti-His monoclonal antibody (1:500) (CWBIO, CW0082) or mixed TB-positive serum (1:200) diluted in TBST. After being washed, PVDF membranes were incubated at $37{ }^{\circ} \mathrm{C}$ for $1 \mathrm{~h}$ with HRPconjugated goat anti-mouse IgG (1:10000) (Abcam, ab6728) or HRP-conjugated anti-human IgG (1:5000) (Abcam, ab6728), respectively. In the end, PVDF membranes was developed with electrochemiluminescence (ECL) using a chemiluminescence imaging system.

\section{ELISA}

The indirect ELISA was used to detect the reactivities between the recombinant RK and serum from patients suffered from TB or healthy human according to previous study (Chen et al. 2018). The 96-well plates were coated with $100 \mu \mathrm{L}$ recombinant $\mathrm{RK}(10 \mu \mathrm{g} / \mathrm{mL})$ diluted with $0.1 \mathrm{M}$ carbonate ( $\mathrm{pH}$ 9.6) per well overnight at $4{ }^{\circ} \mathrm{C}$. The plates were blocked for $2 \mathrm{~h}$ at room temperature with $200 \mu \mathrm{L} \mathrm{5 \%}(w / v)$ skim milk diluted in PBST. One hundred microliters of serum samples diluted 1:500 with PBST were added to each well and incubated $2 \mathrm{~h}$ at $37^{\circ} \mathrm{C}$ followed by three times of washes with PBST. One hundred microliters of HRP-conjugated goat anti-human IgG (Abcam, ab6728) diluted 1:6000 was added to each well and incubated for $1 \mathrm{~h}$ at $37{ }^{\circ} \mathrm{C}$. Finally, chromogenic peroxidase substrate and tetramethylbenzidine (TMB) was added to each well and the reaction was stopped after incubation for $15 \mathrm{~min}$ at $37^{\circ} \mathrm{C}$ in darkness by adding $2 \mathrm{M}$ sulfuric acid. The absorbance was read at $450 \mathrm{~nm}$ using a microplate reader (Tecan, Infinite F50, Swiss).

\section{Statistical analysis}

GRAPHPAD PRISM 5 software was used to analyze the data. The cut-off values were presented as the geometric mean OD for the TB-negative individual serum samples plus $2 \mathrm{SD}$ (standard deviation). Specificity and sensitivity were calculated according to the following formulas: specificity $=$ number of true negatives/(number of true negatives + number of false positives) and sensitivity $=$ number of true positives/(number of true positive + number of false negatives). Difference was statistically significant at $P$ values $<0.05$. The area under the receiver operating characteristic (ROC) curve (AUC) between 0 and 1 indicates the credibility of the ELISA results.

\section{Results}

\section{T7 phage displayed $M$. tuberculosis genomic DNA library was successfully constructed}

T7 phage displayed M. tuberculosis genomic DNA library was constructed in order to screen the M. tuberculosis antigens that specifically bind to the TB-positive serum from the whole genome of M. tuberculosis. As shown in Fig. 1a, there were clear bands near the wells, which suggested that M. tuberculosis genomic DNA was successfully extracted. The genomic DNA was mainly shown as a diffuse band after being digested with Eco RI and Hind III (Fig. 1b). Fragmented M. tuberculosis genomic DNA fragments longer than 200 bp were isolated as shown in Fig. 1c. All 16 plaques randomly selected were successfully amplified by PCR (Fig. 1d), which demonstrated the library has high recombination rate. The exogenous DNA fragments inserted into phages were all within the range of 250-2000 bp, which indicated M. tuberculosis genomic DNA library has good randomness. Results of BLAST analysis showed that these inserted sequences were all derived from the genome DNA of M. tuberculosis. Above these results demonstrated that the T7 phage displayed M. tuberculosis genome DNA library was successfully constructed. The titer of the constructed T7 phage display genomic DNA library was about $6 \times 10^{6} \mathrm{pfu} /$ $\mathrm{cm}^{3}$.This laid the foundation for the screening of dominant antigens from genome-wide of M. tuberculosis.

\section{The TB-positive serum specific phages were successfully enriched}

In order to enrich the phages with good binding specificity to TB-positive serum, three rounds of biopanning were performed for T7 phage displayed genome DNA library of M. tuberculosis. As listed in Table 1, the productivity rate of the first round of biopanning was $2.67 \times 10^{-8}$, while it reached $3.13 \times 10^{-6}$ for the third round of biopanning, which 


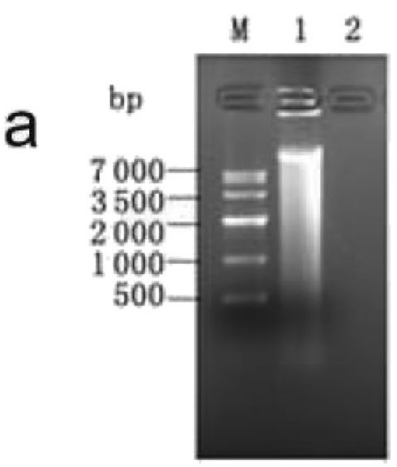

C

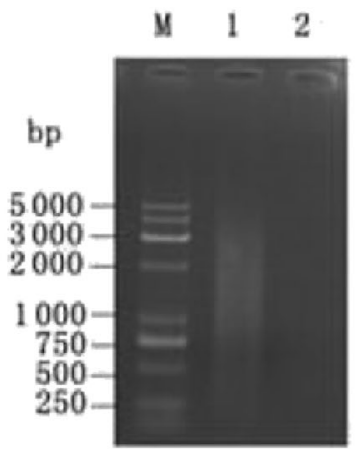

Fig. 1 Construction of the M. tuberculosis T7 phage displayed genomic DNA library. a Agarose gel electrophoresis of genomic DNA of M. tuberculosis. M, DNA marker; 1, genomic DNA of M. tuberculosis; 2, blank control; $\mathbf{b}$ fragmentation of genomic DNA of M. tuberculosis. M, DNA marker; 1 , fragments of M. tuberculosis genomic DNA; $\mathbf{c}$ isolation

demonstrated phage clones bound specifically with TBpositive serum was enriched significantly.

\section{Analysis of the DNA sequences}

In order to analyze the foreign DNA fragments inserted into phages, PCR was performed to amplify the exogenous DNA of the enriched phages challenged from the third rounds of biopanning. The corresponding exogenous DNAs of 19 selected randomly phages were successfully amplified by PCR. The results of BLAST analysis showed that there were four different types for exogenous sequences of 19 phages, namely, representative phages P1-P4, which encoded RK,

Table 1. The ratio of output and input by affinity screening

\begin{tabular}{llll}
\hline Screening rounds & First & Second & Third \\
\hline Input (pfu) & $3.0 \times 10^{11}$ & $3.0 \times 10^{11}$ & $3.0 \times 10^{11}$ \\
Output (pfu) & $8.0 \times 10^{3}$ & $7.1 \times 10^{4}$ & $9.4 \times 10^{5}$ \\
Productivity (\%) & $2.67 \times 10^{-8}$ & $2.37 \times 10^{-7}$ & $3.13 \times 10^{-6}$ \\
\hline
\end{tabular}

Three rounds of biopanning targeted to mixed TB-positive serum and two rounds of negative biopanning were performed for $\mathrm{T} 7$ phage displayed genomic DNA library of $M$. tuberculosis to enrich the phages with high binding specificity to TB-positive serum, and the ratios of output and input were calculated, respectively

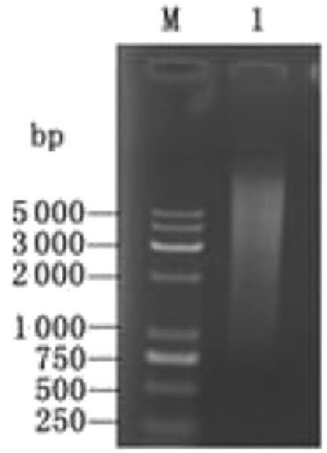

\section{$\begin{array}{llllllllllllll}3 & 4 & 5 & 6 & 7 & 8 & 9 & 10 & 11 & 12 & 13 & 14 & 15 & 16\end{array}$} isolated and purified fragments of genomic DNA; 2, blank control; d agarose gel electrophoresis of PCR products of random clones from M. tuberculosis genomic DNA library. M, DNA maker; 1-16, PCR products

fatty acyl-AMP ligase, cystathionine beta-lyase, and hypothetical protein, respectively. The occurrence frequency of $\mathrm{P} 1$ was highest $(70 \%)$ and the 153 bp DNA sequence inserted P1 phage was $100 \%$ homologous with the DNA sequence of the $436-588$ of the gene coded RK. The locus tag, protein ID, coded protein, matched sequence range, homology, and repeated times of representative phages were listed in Table 2 . Above these results indicated that the four proteins may be TB-positive serum specific binding proteins and RK may be M. tuberculosis dominant antigen challenged from $\mathrm{T} 7$ phage displayed whole-genome DNA library of $M$. tuberculosis.

\section{Representative phages can specifically bind to TB-positive serum}

To identify whether representative phages could bind specifically with mixed TB-positive serum, the immunoreactivity of representative phages were assessed by phage ELISA and dot immunobinding assay, respectively. ELISA results showed that the OD value for the four representative phages were significant higher than the negative serum and control group $(p<0.05)$, which demonstrated these representative phages had good reactivities with TB-positive serum (Fig. 2). For the results of dot immunobinding assay, as shown in the Fig. 3 , representative phages and positive control groups showed 
Table 2 Clustering of the consensus sequences to the inserted sequences of phages

\begin{tabular}{llllll}
\hline Number & Locus_tag & Protein ID & Coded protein & $\begin{array}{l}\text { Matched sequence } \\
\text { range }\end{array}$ & $\begin{array}{c}\text { Homology } \\
\text { times }\end{array}$ \\
\hline P1 & LH57_12000 & AIR14956.1 & Ribokinase & $436-588$ bp & $100 \%$ \\
P2 & B0W96_21030 & AWY82890.1 & Fatty acyl-AMP ligase & $1-204$ bp & $100 \%$ \\
P3 & RV2294 & NP_216810.1 & Cystathionine beta-lyase & $127-216$ bp & $100 \%$ \\
P4 & B0W96_16980 & AWY82212.1 & Hypothetical protein & $1-219$ bp & $100 \%$ \\
Total & & & & & 2 \\
\hline
\end{tabular}

The inserted exogenous DNA sequences of 19 selected phage clones were aligned and analyzed. The inserts from these phages distinguished four representative pages (P1-P4). The locus tag, protein ID, coded protein, matched sequence range, homology, and repeated times of representative phages were listed in Table 2

clear spots, while no spots appeared for the negative control group, which further indicated that these representative phages could bind specifically to the TB-positive serum. Above results demonstrated that RK displayed on the surface of phages can specifically recognize TB-positive serum.

\section{Recombinant RK was successfully expressed and purified}

Due to the highest occurrence frequency of the representative phage P1 whose exogenous DNA coded RK, the recombinant RK was expressed and purified in order to evaluate the potential of RK in the serodiagnosis of TB. The target gene was amplified from genomic DNA of M. tuberculosis H37Rv strain and the recombinant prokaryotic vector $p E T-28 a(+)$ $R K$ was successfully constructed according to the results of PCR identification (Fig. 4a). As shown in Fig. 4b, the recombinant protein was successfully expressed as His-tagged fusion proteins $(36 \mathrm{kDa})$ and then purified using $\mathrm{Ni}-$ nitrilotriacetic acid (NTA) beads. As shown in Fig. 4c,

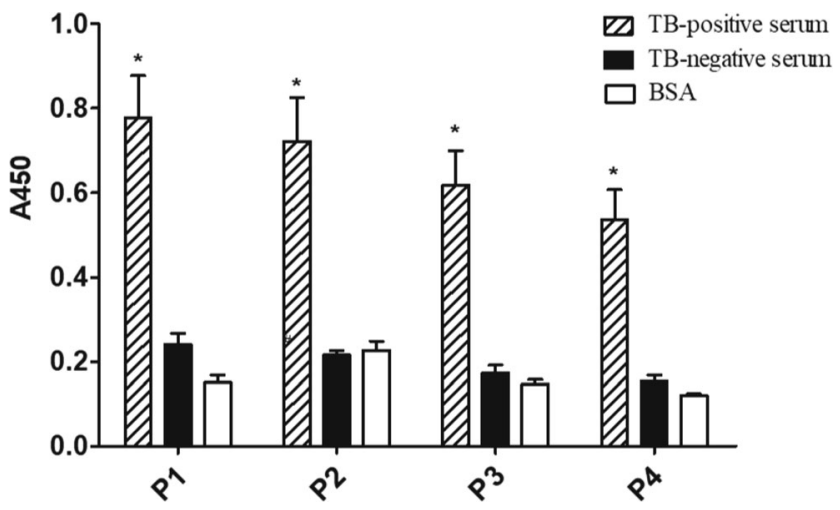

Fig. 2 ELISA analysis of the four representative phages with TBpositive/negative serum and BSA. The ELISA plates were coated with TB-positive serum, negative serum, or BSA, respectively. The four representative phages were added and incubated $2 \mathrm{~h}$ at $37{ }^{\circ} \mathrm{C}$ after blocking. And then HRP-labeled anti-T7 p III monoclonal antibody was added and incubated for $15 \mathrm{~min}$ at $37^{\circ} \mathrm{C}$. The $\mathrm{OD}_{450}$ value was measured. All the assays were carried out in triplicate and the error bars indicate standard deviations. P1-P4 the four representative phages. ${ }^{*} p<0.05$, compared with TB-negative serum or BSA purified RK appeared as a single band via SDS-PAGE analysis. These results indicated that the recombinant RK was successfully expressed and purified.

\section{Identification of recombinant RK by Western blot}

To further identify the target protein and verify that RK could react with different clinical serum, Western blot were performed using mice anti-His antibody, mixed TB-positive serum and TB-negative serum, respectively. The results demonstrated that RK could recognize mice anti-His antibody, which showed that this protein is the recombinant fusion protein. And RK could recognize specially the mixed TB-positive serum, but not reacted with TB-negative serum (Fig. 5), which demonstrated that RK had good reactivity with TB-positive serum.

\section{RK had high sensitivities and specificities for serodiagnosis of TB}

The sensitivities and specificities of RK for the diagnosis of TB were evaluated by indirect ELISA. As shown in Fig. 6a,

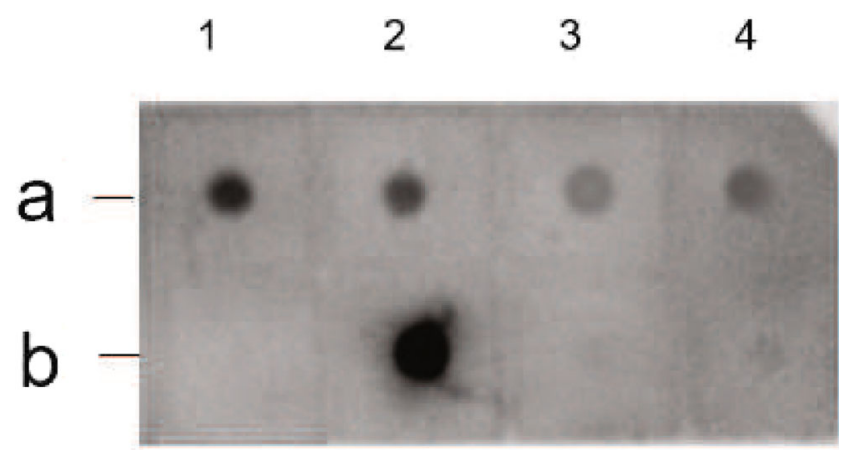

Fig. 3 Dot immunobinding assay of representative phages. The representative phages were added on the small square of the PVDF membrane. The PVDF membrane was then incubated with the purified TB-positive serum, BSA, TB-negative serum, and wild type T7 phage were used as negative controls. a1-a4, representative phages P1-P4; b1, TB-negative serum control; b2, purified TB-positive serum control; b3, wild type $\mathrm{T} 7$ phage control; b4, blank control 


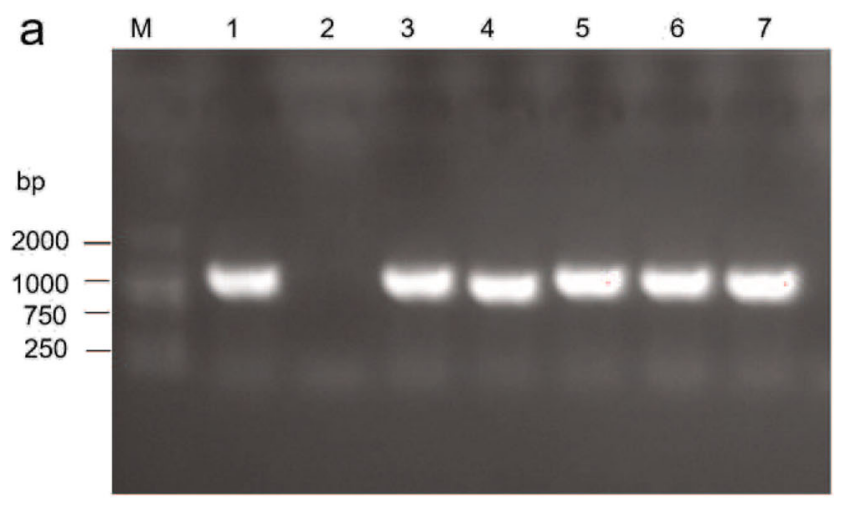

b

C

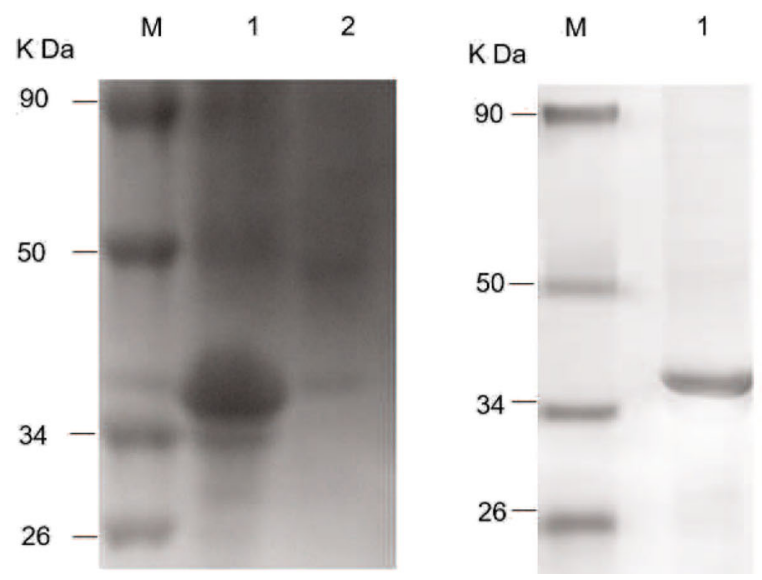

Fig. 4 Expression and purification of recombinant RK. a Identification of the recombinant bacterium containing $p E T-28 a(+)-R K$ by PCR. M, DNA marker; 1-7, expressed bacterium; b SDS-PAGE analysis of the expressed recombinant RK; M, protein marker; 1, IPTG-induced recombinant bacteria with $p E T-28 a(+)-R K ; 2$, non-induced bacteria with $p E T$ $28 a(+)-R K$; $\mathbf{c}$ the purification of the recombinant proteins. Analyses of purified RK using SDS-PAGE were performed as described in Materials and methods. M, protein marker; 1 , the purified target protein

the recombinant $\mathrm{RK}$ could react with most of the serum from TB-positive patients but with only a few TB-negative serum. And the sensitivity and specificity of RK was $90 \%$ and $86 \%$, respectively, which demonstrated RK had high sensitivity and specificity for the serodiagnosis of TB. The statistical analysis including sensitivities, specificities, AUC, cut-off value, and $P$ value were described in Fig. 6b. These data suggested that RK had good potential for the serological diagnosis of TB.

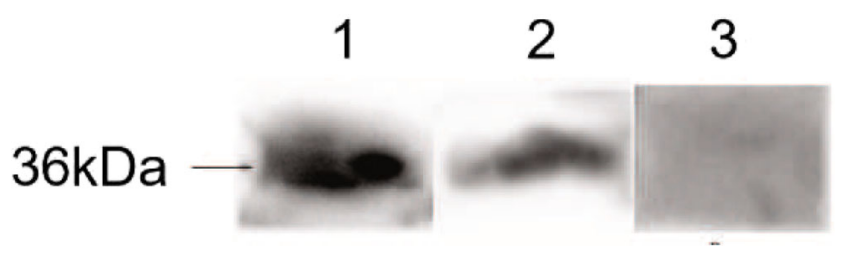

Fig. 5 Western blot analysis of recombinant protein. 1, anti-His antibody; 2, TB-positive serum; 3, TB-negative serum

\section{Discussion}

TB is a major public health problem worldwide ranking above the human immunodeficiency virus (HIV) infection as the leading cause of deaths from infectious diseases (Osei et al. 2017). Accurate and timely identification for infected individuals is critical to the treatment and control of TB. Rapid and early diagnosis of TB and initiating optimal treatment would reduce numbers of TB cases. The lack of effective, sensitive, and specific diagnostic methods was one of the main problems for the prevention and control of TB. Serological diagnosis was widely used in the early diagnosis of TB because of its simplicity, convenience, low cost, high sensitivity, and specificity (Abebe et al. 2007). So far, many specific antigens of M. tuberculosis have been estimated and used for serological diagnosis. However, the specificity and sensitivity were not ideal. Most researchers have studied the antigenicity of a certain protein by expressing and purifying the protein, which wastes time and its operating is cumbersome. Fortunately, it is promising to screen the dominant antigen specifically binding to TB-positive serum from the $M$. tuberculosis wholegenome wide of $M$. tuberculosis by constructing a genomic DNA library and then performing biopanning using TBpositive serum as target molecule.

Phage display technology has been widely used in the field of dominant antigen or epitope screening (Domina et al. 2016), protein-protein interaction, ligand-receptor research, vaccine development, diagnosis, and treatment of cancer because of its advantages such as high-throughput panning and easy purification (Ebrahimizadeh and Rajabibazl 2014). The most commonly used vectors for phage displayed were M13 filamentous phage and T7 phage. As far as the construction of phage displayed genomic DNA library was concerned, T7 phage had obvious advantages as follows: (1) T7 phage is highly stable in various extreme environments such as (high temperature and low pH) (Piggott and Karuso 2016). (2) The life cycle of $\mathrm{T} 7$ phage is shorter so that the process of cloning is more rapid than that of M13 phage (Talwar et al. 2016). Various studies have demonstrated the power of T7 phage displayed in many ways. Talwar et al. (2015) developed a T7 phage displayed library to detect sarcoidosis and TB by a panel of novel antigens. San Segundo-Acosta et al. (2018) identified allergenic peptides and mimotopes from olive pollen and mustard seeds using T7 phage displayed library. Cytidine-5-triphosphate synthase1-selective inhibitory peptide from random peptide library displayed on $\mathrm{T} 7$ phage was discovered by Sakamoto et al. (2017). The specific M. tuberculosis antigens screened from $\mathrm{T} 7$ phage displayed cDNA library may aid to develop a TB vaccine which could generate specific humoral response against $M$. tuberculosis (Talwar et al. 2016). Talwar H et al. developed a T7 phage cDNA library and constructed a microarray platform using sarcoidosis tissue to develop TB therapeutic or prophylactic 


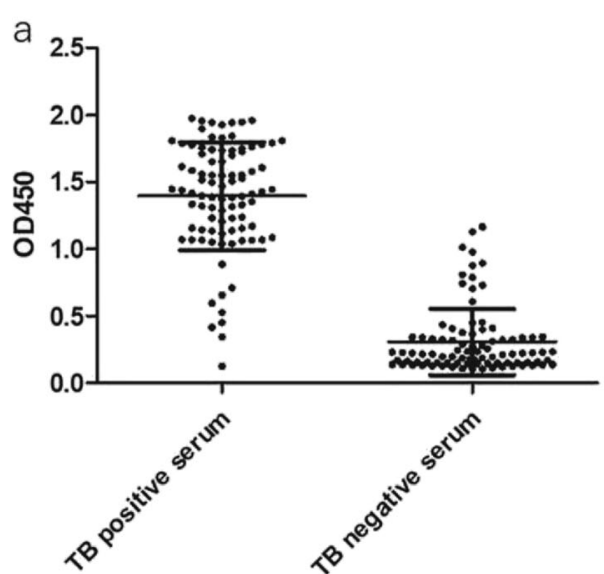

Fig. 6 Reactivities of serum from clinical individuals to recombinant RK and the corresponding ROC curve. ELISA reactivities of RK with different clinical serum and the receiver-operating characteristic (ROC) curve were evaluated with the panel of different serum samples. a ELISA analysis of RK with different clinical serum. ELISA experiments were performed as described in Methods. b The ROC curves of recombinant

vaccines (Talwar et al. 2018). This was different from our study that the potential diagnostic antigen was screened from T7 phage displayed genomic DNA Library of M. tuberculosis.

In this study, four different proteins that could bind specially with TB-positive serum were successfully screened from T7 phage displayed genomic DNA Library of M. tuberculosis. The occurrence frequency of representative phage P1 that displayed exogenous RK was highest. And the results of ELISA and dot immunobinding assay showed that RK had highest binding specificity to the TB-positive serum. Highly specific phage clones could be screened in this study due to the reverse adsorption using TB-negative serum and blank microplate, as well as decreasing target protein concentration, increasing the elution solution concentration, reducing the binding time and prolonging the elution time gradually during the second and third biopanning. For the further studies, RK was successfully expressed and purified and the reactivities of recombinant RK with different clinical serum was detected. ELISA results showed that RK had high specificity and sensitivity in the serodiagnosis of TB.

RK genes have been found in both prokaryotes and eukaryotes, and sequence comparisons have shown that they belong to the PfkB family of carbohydrate kinases, which is also referred to the RK family (Park and Gupta 2008). A RK gene from the anaerobic halothermophilic bacterium Halothermothrix orenii was cloned and overexpressed in E. coli by Kori et al. (2012). Yang Q et al. have characterized the activities of two putative RKs, Rv2436 and Ms4585, from M. tuberculosis and M. smegmatis, respectively (Yang et al.2011). And study proved that RK could physically interact with the mycobacterial topoisomerase I (Top A) both in vivo and in vitro. However, the function and regulation of RKs in M. tuberculosis are still largely unknown.

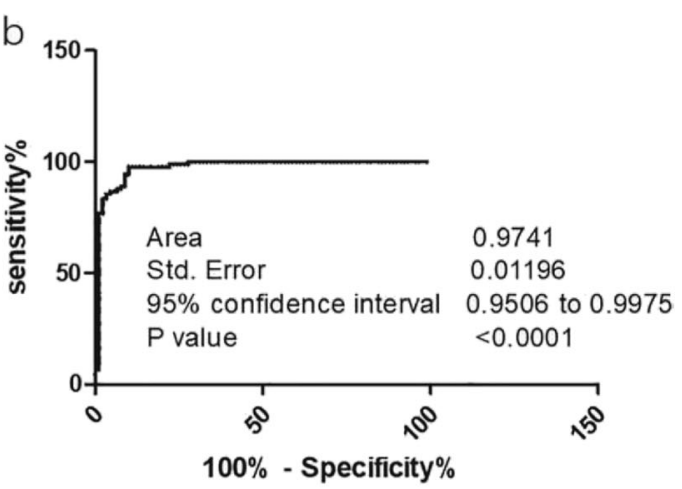

RK. The corresponding ROC curves for recombinant RK were produced using GRAPHPAD PRISM 5 according to the distribution of the optical density in ELISA test. And the area under the curve (AUC), standard error (SE), sensitivity, and specificity with $95 \%$ confidence intervals (95\% CI) and $P$ value were indicated

In a word, our study involved the construction of $\mathrm{T} 7$ phage displayed M. tuberculosis genomic DNA library by using T7 phage as a carrier, which was subsequently screened for the dominant $M$. tuberculosis antigen recognized specially with the TB-positive serum. We assessed RK exhibited high sensitivity (90\%) and high specificity $(86 \%)$ for the detection of $M$. tuberculosis infection. Our findings demonstrated that RK could be used as a novel perspective antigen for the development of M. tuberculosis diagnostic assays. However, there was a disadvantage due to the limitations of the sample source, that is to say, all TB-positive serum was from patients with active TB. In order to further evaluate the application value of RK in the serodiagnosis of TB, we will further expand serum samples to analyze all samples from patients including non-active TB. For the further studies, we will express and purify the three proteins displayed by the other three representative phages and then evaluate the potential value of these recombinant proteins in the serological diagnosis of TB.

Funding information This study was funded by "Prevention and Control of AIDS and Viral Hepatitis etc. Other Major Infectious Disease"- - the Science and Technology Major Special Project of the 12th Five-Year Plan of China (grant number 10003006-002) and the National Natural Science Foundation of China (grant number 81871256).

\section{Compliance with ethical standards}

Conflict of interest The authors declare that they have no conflict of interest.

Ethical statement The study was approved by the ethics review board of University of South China. 


\section{References}

Abebe F, Holm-Hansen C, Wiker HG, Bjune G (2007) Progress in serodiagnosis of Mycobacterium tuberculosis infection. Scand J Immunol 66(2-3):176-191

Baumann R, Kaempfer S, Chegou NN, Oehlmann W, Loxton AG, Kaufmann SH, van Helden PD, Black GF, Singh M, Walzl G (2014) Serologic diagnosis of tuberculosis by combining classes against selected mycobacterial targets. J Inf Secur 69:581-589

Beghetto E, De Paolis F, Montagnani F, Cellesi C, Gargano N (2009) Discovery of new Mycoplasma pneumoniae antigens by use of a whole-genome lambda display library. Microbes Infect 11(1):66-73

Chen Y, Cao S, Liu Y, Zhang X, Wang W, Li C (2018) Potential role for Rv2026c- and Rv2421c- specific antibody responses in diagnosing active tuberculosis. Clin Chim Acta 487:369-376

De Paolis F, Beghetto E, Spadoni A, Montagnani F, Felici F, Oggioni MR, Gargano N (2007) Identification of a human immunodominant B-cell epitope within the immunoglobulin A1 protease of Streptococcus pneumoniae. BMC Microbiol 7:113

Deng X, Wang L, You X, Dai P, Zeng Y (2018) Advances in the T7 phage display system (review). Mol Med Rep 17:714-720

Di Cristina M, Del Porto P, Buffolano W, Beghetto E, Spadoni A, Guglietta S, Piccolella E, Felici F, Gargano N (2004) The Toxoplasma gondii bradyzoite antigens BAG1 and MAG1 induce early humoral and cell-mediated immune responses upon human infection. Microbes Infect 6:164-171

Domina M, Lanza Cariccio V, Benfatto S, Venza M, Venza I, Borgogni E, Castellino F, Midiri A, Galbo R, Romeo L, Biondo C, Masignani V, Teti G, Felici F, Beninati C (2016) Functional characterization of a monoclonal antibody epitope using a lambda phage displayed-deep sequencing platform. Sci Rep 6:31458

Ebrahimizadeh W, Rajabibazl M (2014) Bacteriophage vehicles for phage display: biology, mechanism, and application. Curr Microbiol 69:109-120

Kori LD, Hofmann A, Patel BK (2012) Expression, purification, crystallization and preliminary $\mathrm{X}$-ray diffraction analysis of a ribokinase from the thermohalophile Halothermothrix orenii. Acta Crystallogr Sect F Struct Biol Cryst Commun 68:240-243

Krishna P, Jain A, Bisen PS (2016) Microbiome diversity in the sputum of patients with pulmonary tuberculosis. Eur J Clin Microbiol Infect Dis 35:1205-1215

Luo L, Zhu L, Yue J, Liu J, Liu G, Zhang X, Wang H, Xu Y (2017) Antigens Rv0310c and Rv1255c are promising novel biomarkers for the diagnosis of Mycobacterium tuberculosis infection. Emerg Microbes Infect 6:e64

Machado D, Couto I, Viveiros M (2018) A rapid molecular assays for detection of tuberculosis. Pneumonia (Nathan) 10(1):4

Osei E, Der J, Owusu R, Kofie P, Axame WK (2017) The burden of HIV on Tuberculosis patients in the Volta region of Ghana from 2012 to 2015: implication for Tuberculosis control. BMC Infect Dis 17:504

Park J, Gupta RS (2008) Adenosine kinase and ribokinase- the RK family of proteins. Cell Mol Life Sci 65:2875-2896

Piggott AM, Karuso P (2016) Identifying the cellular targets of natural products using T7 phage display. Nat Prod Rep 33:626-636

Qiang X, Sun K, Xing L, Xu Y (2017) Discovery of a polystyrene binding peptide isolated from phage displayed library and its application in peptide immobilization. Sci Rep 7:2673

Roupie V, Alonso-Velasco E, Van Der Heyden S, Holbert S, Duytschaever L, Berthon P, Van Dosselaer I, Van Campe W, Mostin L, Biet F, Roels S, Huygen K, Fretin D (2018) Evaluation of mycobacteria-specific gamma interferon and antibody responses before and after a single intradermal skin test in cattle naturally exposed to M. avium subsp. paratuberculosis and experimentally infected with M. bovis. Vet Immunol Immunopathol 196:35-47
Sakamoto K, Kawata Y, Masuda Y, Umemoto T, Ito T, Asami T, Takekawa S, Ohtaki T, Inooka H (2016) Discovery of an artificial peptide agonist to the fibroblast growth factor receptor $1 \mathrm{c} / \beta \mathrm{klotho}$ complex from random peptide T7 phage display. Biochem Biophys Res Commun 480:55-60

Sakamoto K, Ishibashi Y, Adachi R, Matsumoto SI, Oki H, Kamada Y, Sogabe S, Zama Y, Sakamoto JI, Tani A (2017) Identification of cytidine-5-triphosphate synthase1-selective inhibitory peptide from random peptide library displayed on T7 phage. Peptides 94: $56-63$

San Segundo-Acosta P, Garranzo-Asensio M, Oeo-Santos C, MonteroCalle A, Quiralte J, Cuesta-Herranz J, Villalba M, Barderas R (2018) High-throughput screening of T7 phage display and protein microarrays as a methodological approach for the identification of IgEreactive components. J Immunol Methods 456:44-53

Shi W, Zhao L, Li S, Xu G, Zeng Y (2018) Serological diagnosis of mycoplasma pneumoniae infection by using the mimic epitopes. World J Microbiol Biotechnol 34:82

Talwar H, Rosati R, Li J, Kissner D, Ghosh S, Madrid FF, Samavati L (2015) Development of a T7 phage display library to detect sarcoidosis and tuberculosis by a panel of novel antigens. Ebio medicine 2: $341-350$

Talwar H, Talreja J, Samavati L (2016) T7 phage display library a promising strategy to detect tuberculosis specific biomarkers. Mycobact Dis 6:214

Talwar H, Hanoudi SN, Draghici S, Samavati L (2018) Novel T7 phage display library detects classifiers for active Mycobacterium tuberculosis infection. Viruses 10:375

Theron G, Peter J, van Zyl-Smit R, Mishra H, Streicher E, Murray S, Dawson R, Whitelaw A, Hoelscher M, Sharma S, Pai M, Warren R, Dheda K (2011) Evaluation of the Xpert MTB/RIF assay for the diagnosis of pulmonary tuberculosis in a high HIV prevalence setting. Am J Respir Crit Care Med 184:132-172

Wang Y, Wang L (2017) Vaccination with phage-displayed antigenic epitope. Methods Mol Biol 1625:225-235

Wang L, Deng X, Liu H, Zhao L, You X, Dai P, Wan K, Zeng Y (2016) The mimic epitopes of Mycobacterium tuberculosis screened by phage displayed peptide library have serodiagnostic potential for tuberculosis. Pathoge Dis 74. https://doi.org/10.1093/femspd/ ftw091

$\mathrm{Xu}$ JN, Chen JP, Chen DL (2012) Serodiagnsis efficacy and immunogenicity of the fusion protein of Mycobacterium tuberculosis composed of the 10-kilodalton culture filtrate protein, ESAT-6, and the extracellular domain fragment of PPE68. Clin Vaccine Immunol 19(4):536-580

Yan ZH, Yi L, Wei PJ, Jia HY, Wang J, Wang XJ, Yang B, Gao X, Zhao YL, Zhang HT (2018) Evaluation of panels of Mycobacterium tuberculosis antigens for serodiagnosis of tuberculosis. Int $\mathrm{J}$ Tuberc Lung Dis 22(8):959-965

Yang Q, Liu Y, Huang F, He ZG (2011) Physical and functional interaction between D-ribokinase and topoisomerase I has opposite effects on their respective activity in Mycobacterium smegmatis and Mycobacterium tuberculosis. Arch Biochem Biophys 512(2):135142

You X, Li R, Wan K, Liu L, Xie X, Zhao L, Wu N, Deng X, Wang L, Zeng Y (2017) Evaluation of Rv0220, Rv2958c, Rv2994 and $\mathrm{Rv} 3347 \mathrm{c}$ of Mycobacterium tuberculosis for serodiagnosis of tuberculosis. Microb Biotechnol 10:604-611

Zhou F, Xu X, Wu S, Cui X, Fan L, Pan W (2015) Protein array identification of protein markers for serodiagnosis of Mycobacterium tuberculosis infection. Sci Rep 5:15349

Publisher's note Springer Nature remains neutral with regard to jurisdictional claims in published maps and institutional affiliations. 